\title{
Dynamic compressive behaviour of sandwich panels with lattice truss core filled by shear thickening fluid
}

\author{
Z.P. Gu ${ }^{\mathrm{a}, \mathrm{b}}$, X.Q. Wu $\mathrm{Wu}^{\mathrm{a}, \mathrm{e}, *}$, Q.M. $\mathrm{Li}^{\mathrm{c}}$, Q.Y. Yin ${ }^{\mathrm{d}, \mathrm{f}}$, C.G. Huang ${ }^{\mathrm{a}, \mathrm{b}}$ \\ ${ }^{a}$ Key Laboratory of Mechanics in Fluid Solid Coupling Systems, Institute of Mechanics, Chinese Academy of Sciences, Beijing 100190, China \\ ${ }^{\mathrm{b}}$ School of Engineering Science, University of Chinese Academy of Sciences, Beijing 100049, China \\ ${ }^{\mathrm{c}}$ Department of Mechanical, Aerospace and Civil Engineering, School of Engineering, The University of Manchester, Manchester M13 9PL, UK \\ d Department of Applied Mechanics and Engineering, School of Engineering, Sun Yat-sen University, Guangzhou 510275, China \\ ${ }^{\mathrm{e}}$ Materials and Process Simulation Center, California Institute of Technology, Pasadena, CA 91125, UK \\ ${ }^{\mathrm{f}}$ State Key Laboratory for Strength and Vibration of Mechanical Structures, Xi'an Jiaotong University, 710049, China
}

\section{A R T I C L E I N F O}

\section{Keywords:}

Dynamic compressive behaviour

Sandwich panels with lattice truss core

Shear thickening fluid

Hydrodynamic constitutive model

Analytical model

Fluid-structure interaction

\begin{abstract}
A B S T R A C T
The compressive behaviour of sandwich panels with lattice truss core filled by shear thickening fluid (SPLTCSTF) at high strain-rates is performed analytically and numerically. Firstly, a hydrodynamic constitutive model for the shear thickening fluid (STF) involving shear thinning, shear thickening, and hydrostatic compressibility is undertaken to describe the dynamic behaviour of the STF. Then an analytical model based on the squeezing flow of viscous fluids is proposed. The squeezing resistance of the STF between the two panels of the SPLTC under various loading velocities is analysed using a fluid-structure interaction (FSI) simulation, by which the constitutive parameters of the STF are obtained. Finally, the dynamic response of the SPLTC-STF involving buckling and post-buckling of core struts in the STF is investigated using the FSI method. The enhanced energy absorption capacity of the SPLTC-STF observed in Ref [1] is numerically interpreted. The effects of shear thickening behaviour of STF on the dynamic response of SPLTC-STF are predicted, providing a method of optimal design for STF filled sandwich panels over a wide range of impulse loadings for dynamic energy absorption.
\end{abstract}

\section{Introduction}

Structural materials can be designed to realize various functions such as high ballistic limit and shock absorption. As one kind of advanced structural material, sandwich panels with lattice truss core (SPLTC) have attracted significant interest in the research community [2-4]. There are considerable publications that address the mechanical performance of SPLTC [5-11], showing that the SPLTC can dissipate dynamic energy by various deformation modes. However, as a protective structure, the usage of SPLTC is still hindered by its unsatisfied dynamic energy absorption capacity and rapid degradation of stiffness during impact $[3,7,12]$.

Filling with lightweight materials has been proved to be a practical solution to enhance the impact resistance and energy absorption of the SPLTC. Many types of fillers such as low-density polymers and ceramics have been considered to improve the impact protection capacity of SPLTC $[13,14]$. Shear thickening fluid (STF), as an intelligent material, is characterized by its reversible energy absorption behaviour under impulse loading [15-18] and remarkable energy absorption capacity by means of viscous dissipation during shear thickening [16, 19-21] and compression thickening [22, 23]. The vibration attenuation [24-26] and penetration protection [27, 28] properties of sandwich structures filled with STF have been measured by Gurgen et al. [24, 27], Fischer et al. [25], and Tan et al. [28] The results showed that the shock absorption and stab resistance capacity of the sandwich structures have been greatly improved after adding STF. Recently, the dynamic behaviour of the SPLTC-STF has been measured [1]. The results showed that the dynamic compressive strength of the SPLTC-STF increases significantly when compared to that of the SPLTC. In addition, the study in the previous experiments [1] revealed the enhanced dynamic energy absorption behaviour of the SPLTC-STF due to their coupling enhancement effect under impulsive loading.

To improve the impact resistance of SPLTC-STF, it requires further understanding of the coupling mechanisms of energy dissipation between SPLTC and STF under impulsive loadings. Therefore, it is important to model the compression behaviour of the SPLTC-STF. Successful analytical and numerical models of empty or solid-filled SPLTC have been proposed, based on which the dynamic response of

\footnotetext{
* Corresponding author.

E-mail address: wuxianqian@imech.ac.cn (X.Q. Wu).
} 
the corresponding sandwich panels could be predicted [11, 29-34]. The fluid-structure interaction (FSI) between the surrounding water and the face sheets of the sandwich panels was also investigated [5, 29, 35]. However, the coupling effect between SPLTC and the inside STF fillers, which is crucial for the understanding of the dynamic response of SPLTC-STF, has not been performed based on the authors' best knowledge due to the lack of hydrodynamic constitutive models for STF. In this paper, the parameters of the hydrodynamic constitutive model for STF are obtained, and the coupling energy dissipation mechanisms of the SPLTC-STF under compression are studied analytically and numerically. The effect of the rheology behaviour of the STF on the impact response of the SPLTC-STF is also investigated.

The paper is organized as follows. In Section 2, a hydrodynamic constitutive model of the STF is described. In Section 3, analytical and numerical models describing the compressive behaviour of the STF are developed. In Section 4, the buckling and post-buckling behaviour of the SPLTC-STF is analysed based on an FSI numerical model, followed by discussions and conclusions.

\section{Hydrodynamic constitutive model of STF}

To perform the modelling and numerical simulation on the compression behaviour of the SPLTC-STF requires an understanding of the dynamic behaviour of STF. Here, a hydrodynamic constitutive model of STF is employed based on the previous experimental results [1, 22, 23] to describe the dynamic shearing and compressive behaviour of the STF.

The STF is a non-Newtonian fluid whose dynamic constitutive equation can be described by a hydrostatic response (equation of state or EOS) and viscous response under shear (deviatoric) stresses [36]. Its dynamic shear viscosity changes significantly with increasing shear strain-rate. Meanwhile, its volumetric compressibility has a non-linear relationship with the hydrostatic pressure. Therefore, the stress applied on the STF, $\sigma_{i j}$, is decomposed into deviatoric stress, $s_{i j}$, and hydrostatic pressure, $p$, i.e.

$\sigma_{i j}=-p \delta_{i j}+s_{i j}$,

where $\delta_{i j}$ is Kronecker delta function.

The corresponding strain, $e_{i j}$, is

$e_{i j}=\frac{1}{3} e_{i i} \delta_{i j}+\varepsilon_{i j}$,

where $e_{i i}$ and $\varepsilon_{i j}$ are volumetric strain and deviatoric strain, respectively.

Accordingly, the strain-rate is

$\frac{d e_{i j}}{d t}=\frac{1}{3 V} \frac{d V}{d t} \delta_{i j}+\frac{d \varepsilon_{i j}}{d t}$,

where $V$ is specific volume.

The dynamic viscosity of the STF [36], $\mu_{i j}$, is a function of the shear strain-rate, $\dot{\varepsilon}_{i j}$,

$\mu_{i j}=m \cdot \dot{\varepsilon}_{i j}^{n-1}$,

where $m$ and $n$ are material constants. Then, the deviatoric stresses are determined by

$s_{i j}=\mu_{i j} \cdot \dot{\varepsilon}_{i j}=m \cdot \dot{\varepsilon}_{i j}^{n}$.

Similar to Petel et al. [37, 38], the Mie-Grüneisen EOS [39-41] is used to describe the hydrostatic behaviour of the STF,

$U_{s}=C_{0}+S \cdot U_{p}$,

where $U_{s}$ and $U_{p}$ are shock velocity and particle velocity, respectively. $C_{0}$ and $S$ are material constants.

In the previous studies [22, 23], the relationship between the shock velocity, $U_{s}$, and particle velocity, $U_{p}$, for the STF has been obtained using laser-induced shock experiments,

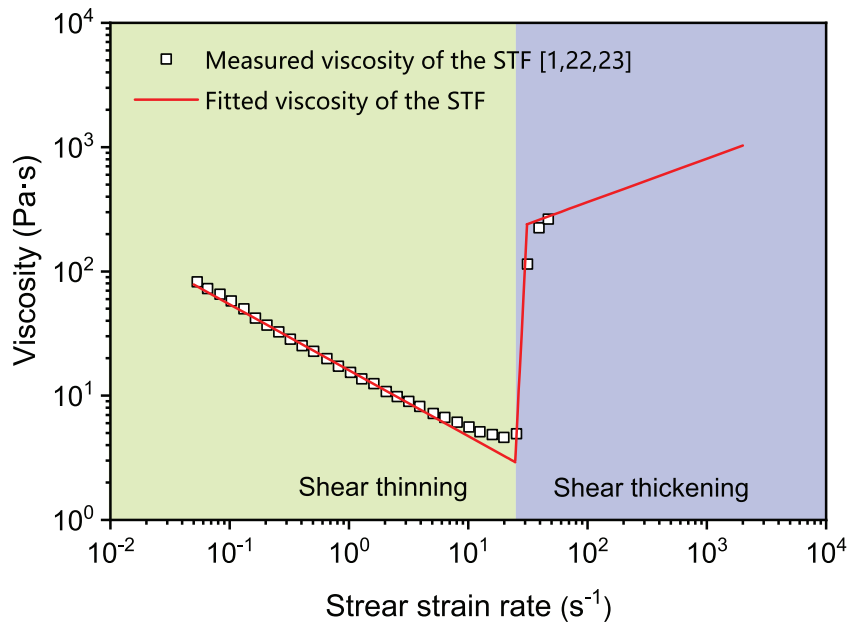

Fig. 1. Shear-thinning and shear thickening behaviour of the STF used in modelling and numerical simulation [1].

$U_{s}=2050+5.324 U_{p}$,

where $U_{s}$ and $U_{p}$ have a unit of $\mathrm{m} / \mathrm{s}$.

The parameters $m$ and $n$ in Eq. (4) can be obtained by fitting experimental results. The steady-state shear viscosity of the 57 vol./vol.\% spherical silica particle-polyethene glycol STF has been measured in the previous study [1]. As shown in Fig. 1, the viscosity of the STF is about $100 \mathrm{~Pa} \cdot \mathrm{s}$ at a shear strain-rate of $5 \times 10^{-2} \mathrm{~s}^{-1}$. With the increase of shear strain-rate, the viscosity of the STF initially decreases gradually, indicating the shear-thinning regime, which is followed by a steep increase at a critical shear strain-rate of about $25 \mathrm{~s}^{-1}$ corresponding to the start of the shear thickening regime. It should be noted that the viscosities of the STF in the shear-thickening regime are not fully measured [1] when the shear strain-rates are higher than $47 \mathrm{~s}^{-1}$ due to the limitation of the rotational rheometer. For the shear-thinning regime, i.e. shear strain-rates are lower than $25 \mathrm{~s}^{-1}$, as shown in Fig. 1, $m=16$ and $n=0.47$. Once the shear thickening happens, the viscosity of the STF increases significantly, and then it is hard to fully obtain the viscosity of the STF in the shear-thickening regime at high shear strainrates. In the present study, the shear strain-rate dependent viscosity of STF in the shear thickening regime is obtained based on the previous experimental result and the FSI simulation, which will be elucidated in detail in the following sections of modelling and numerical analysis.

\section{Modelling of compression resistance of STF}

During the dynamic compression of the SPLTC-STF, the face sheets of the SPLTC squeeze the STF filler. Meanwhile, the core struts buckle symmetrically under the lateral pressure induced by the shear deformation and inertial effects of the surrounding STF, which could be in either shear-thinning or shear-thickening regimes depending on the local deformation state. Therefore, the dynamic compressive stress of the SPLTC-STF could be decomposed physically into the squeezing resistance of the STF and the symmetrical buckling and post-buckling resistance of the core struts. Here, the squeezing flow model for a nonNewtonian fluid layer is developed to describe the squeezing behaviour of the STF. However, as it is difficult to theoretically obtain the transient squeezing resistance of the STF with both shear-thinning and shear-thickening behaviour, the FSI numerical simulation with the hydrodynamic constitutive model and the EOS for the STF is performed using LS-DYNA [42] to analyse the dynamic squeezing response of the STF, from which the constitutive model parameters $m$ and $n$ are determined by calibrating with the experimental results. The plastic postbuckling behaviour of the core struts under the unsteady lateral loadings induced by the nonlinear interaction between the core struts and 


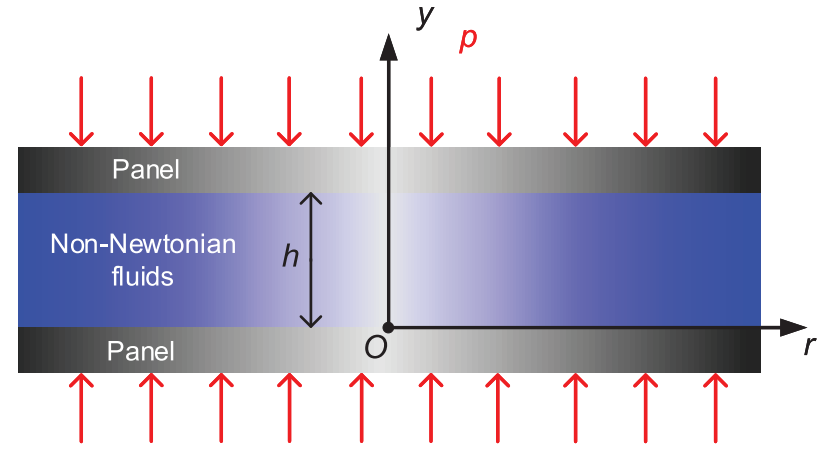

Fig. 2. Squeezing flow model for non-Newtonian fluids [43].

the surrounding STF is analysed by the same FSI numerical simulation method.

\subsection{Analytical squeezing flow model for non-Newtonian fluids}

Suppose that the fluid layer with thickness, $h$, is squeezed by two circular plates with diameters of $2 R$, as shown in Fig. 2.

The movement of the fluid layer is assumed to be a two-dimensional flow, and obeys the following equation [43],

$\frac{\partial^{2} u}{\partial y^{2}}=\frac{1}{\mu} \frac{\partial p}{\partial r}+\frac{\rho}{\mu}\left(\frac{\partial u_{0}}{\partial t}+u_{0} \frac{\partial u_{0}}{\partial r}+v_{0} \frac{\partial u_{0}}{\partial y}\right)-\left(\frac{\partial^{2} u_{0}}{\partial r^{2}}+\frac{1}{r} \frac{\partial u_{0}}{\partial r}-\frac{u_{0}}{r^{2}}\right)$,

where $\mu$ is viscosity of the fluid, $p$ is pressure, $u_{0}$ and $v_{0}$ are solutions of the velocities in $y$ and $r$ directions, respectively, when neglecting the inertial effect of the fluid layer, and

$u_{0}=\frac{3 r \dot{h}}{h^{3}}\left(y^{2}-h y\right), \quad v_{0}=-\frac{\dot{h}}{h^{3}}\left(2 y^{3}-3 h y^{2}\right)$,

where $\dot{h}$ is the squeezing velocity. Substitute Eq. (9) into Eq. (8) to obtain

$\frac{\partial^{2} u}{\partial y^{2}}=\frac{1}{\mu} \frac{\partial p}{\partial r}+\frac{\rho}{\mu}\left(\frac{3 r \ddot{h}}{h^{3}}\left(y^{2}-h y\right)+\frac{r \dot{h}^{2}}{h^{2}}\left(-\frac{3 y^{4}}{h^{4}}+\frac{6 y^{3}}{h^{3}}-\frac{9 y^{2}}{h^{2}}+\frac{6 y}{h}\right)\right)$.

The shear strain-rate of the fluid is

$\dot{\varepsilon}=\frac{\partial u_{0}}{\partial y}+\frac{\partial v_{0}}{\partial r}=\frac{3 r \dot{h}}{h^{3}}(2 y-h)$.

According to Eq. (4), the viscosity of the non-Newtonian fluid is $\mu=m \dot{\varepsilon}^{n-1} \cdot n<1$ and $n>1$ denote the shear thinning and shear thickening behaviours of the fluid, respectively. Consequently,

$\frac{1}{\mu}=\frac{1}{m} \dot{\varepsilon}^{1-n}=\alpha \dot{\varepsilon}^{\beta}=\alpha\left(\frac{3 r \dot{h}}{h^{3}}(2 y-h)\right)^{\beta}$,

where

$\alpha=\frac{1}{m}, \beta=1-n$.

Therefore, Eq. (10) can be rewritten as

$\frac{\partial^{2} u}{\partial y^{2}}=\frac{\partial p}{\partial r} \alpha\left(\frac{3 r \dot{h}}{h^{3}}(2 y-h)\right)^{\beta}$

$+\rho \alpha\left(\frac{3 r \dot{h}}{h^{3}}(2 y-h)\right)^{\beta} \cdot\left(\frac{3 r \ddot{h}}{h^{3}} \cdot\left(y^{2}-h y\right)+\frac{r \dot{h}^{2}}{h^{2}} \cdot\left(-\frac{3 y^{4}}{h^{4}}+\frac{6 y^{3}}{h^{3}}-\frac{9 y^{2}}{h^{2}}+\frac{6 y}{h}\right)\right)$

Define $2 y-h=\xi$. Therefore, $\xi=0, \mu=0 ; \xi=h, \frac{1}{\mu}=\alpha\left(\frac{3 r \dot{h}}{h^{2}}\right)^{\beta}$,

$\frac{1}{\mu}=\alpha\left(\frac{3 r \dot{h}}{h^{3}}(2 y-h)\right)^{\beta}=\alpha\left(\frac{3 r \dot{h}}{h^{3}} \xi\right)^{\beta}$.
Integrating function $u$ with respect to variable $\xi$ and considering boundary conditions $\xi=0, \frac{\partial u}{\partial \xi}=0$ and $\xi=h, u=0$, the following equation is obtained,

$$
\begin{aligned}
& 4 u=\alpha\left(\frac{3 r \dot{h}}{h^{3}}\right)^{\beta} \frac{\partial p}{\partial r}\left(\frac{\xi^{2+\beta}}{(1+\beta)(2+\beta)}+C_{1}\right) \\
& +\rho \alpha\left(\frac{3 r \dot{h}}{h^{3}}\right)^{\beta}\left(\begin{array}{l}
\frac{3 r \ddot{h}}{4 h^{3}}\left(\frac{\xi^{4+\beta}}{(3+\beta)(4+\beta)}-h^{2} \frac{\xi^{2+\beta}}{(1+\beta)(2+\beta)}+C_{2}\right) \\
+\frac{3 r \dot{h}^{2}}{16 h^{2}}\left(-\frac{\xi^{6+\beta}}{h^{4}(5+\beta)(6+\beta)}-\frac{6 \xi^{4+\beta}}{h^{2}(3+\beta)(4+\beta)}+\frac{7 \xi^{2+\beta}}{(1+\beta)(2+\beta)}+C_{3}\right)
\end{array}\right),
\end{aligned}
$$

where

$$
\begin{aligned}
& C_{1}=-\frac{h^{2+\beta}}{(1+\beta)(2+\beta)} \\
& C_{2}=\left(-\frac{1}{(3+\beta)(4+\beta)}+\frac{1}{(1+\beta)(2+\beta)}\right) h^{4+\beta} \\
& C_{3}=\left(\frac{1}{(5+\beta)(6+\beta)} \frac{6}{h^{2}(3+\beta)(4+\beta)}-\frac{7}{(1+\beta)(2+\beta)}\right) h^{2+\beta}
\end{aligned} .
$$

From continuity of deformation,

$\int_{0}^{h} 4 u d \xi=-r \dot{h}$.

Integrating Eq. (17), we obtain

$\int_{0}^{h} 4 u d \xi=\alpha\left(\frac{3 r \dot{h}}{h^{3}}\right)^{\beta} \frac{\partial p}{\partial r} m_{1} h^{3+\beta}+\rho \alpha\left(\frac{3 r \dot{h}}{h^{3}}\right)^{\beta}\left(\frac{3 r \ddot{h}}{h^{3}} m_{2} h^{5+\beta}+\frac{3 r \dot{h}^{2}}{16 h^{2}} m_{3} h^{5+\beta}\right)$,

$=-r \dot{h}$

where

$m_{1}=-\frac{1}{(1+\beta)(3+\beta)}$

$m_{2}=\frac{1}{(1+\beta)(3+\beta)(5+\beta)}$

$m_{3}=\frac{1}{(5+\beta)(7+\beta)}+\frac{6}{(3+\beta)(5+\beta)}-\frac{7}{(1+\beta)(3+\beta)}$

The pressure can be obtained by integrating $p$ with respect to $r$ in Eq. (18),

$p=-\frac{\dot{h}}{a(3 \dot{h})^{\beta} m_{1} h^{3-2 \beta}} \frac{r^{2-\beta}}{2-\beta}-\left(\frac{3 m_{2} \rho \ddot{h}}{4 m_{1} h}+\frac{3 m_{3} \rho \dot{h}^{2}}{16 m_{1} h^{2}}\right) \frac{r^{2}}{2}+C_{4}$,

with boundary condition $p(R, t)=p_{0}$, where $p_{0}$ is the ambient pressure, the constant $C_{4}$ is determined as

$C_{4}=p_{0}+\frac{\dot{h}}{a(3 \dot{h})^{\beta} m_{1} h^{3-2 \beta}} \frac{R^{2-\beta}}{2-\beta}-\left(\frac{3 m_{2} \rho \ddot{h}}{4 m_{1} h}+\frac{3 m_{3} \rho \dot{h}^{2}}{16 m_{1} h^{2}}\right) \frac{R^{2}}{2}$

Then, the squeezing force of the non-Newtonian fluid is given by

$F_{\text {squeeze }}=\int_{0}^{R} 2 \pi r\left(p-p_{0}\right) d r$

$=\frac{\pi \dot{h}}{a(3 \dot{h})^{\beta} m_{1} h^{3-2 \beta}} \frac{R^{4-\beta}}{4-\beta}+\left(\frac{3 m_{2} \rho \ddot{h}}{4 m_{1} h}+\frac{3 m_{3} \rho \dot{h}^{2}}{16 m_{1} h^{2}}\right) \frac{\pi R^{4}}{4}$

The nominal stress versus strain relationships of the non-Newtonian fluid at various compressive strain-rates are determined as

$\sigma_{\text {squeeze }}=\frac{F}{\pi R^{2}}$,

$\dot{\varepsilon}_{\text {squezze }}=\frac{\dot{h}}{h}$,

$\varepsilon_{\text {squeeze }}=\int_{0}^{t} \dot{\varepsilon}_{\text {squeeze }} d t$,

where $\sigma_{\text {squeeze, }} \varepsilon_{\text {squeeze }}$ and $\dot{\varepsilon}_{\text {squeeze }}$ are nominal stress, strain and strain- 


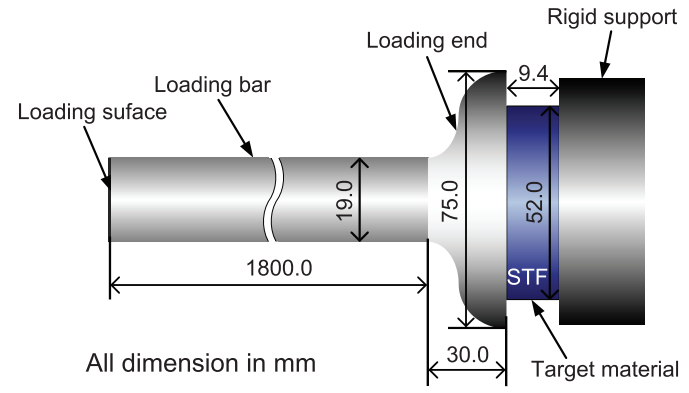

(a)

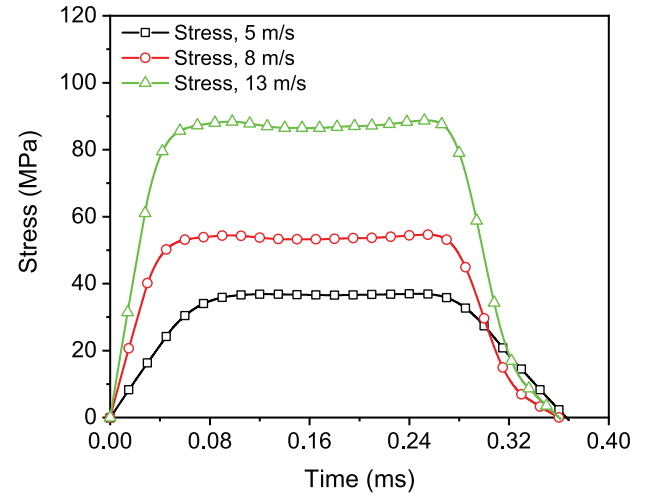

(b)

Fig. 3. Numerical models of (a) schematic, and (b) the loading pressure histories at the impact velocities of 5, 8 , and $13 \mathrm{~m} / \mathrm{s}$.

rate, respectively.

\subsection{Simulation results of squeezing behaviour of STF}

For non-Newton fluids with only shear-thinning or shear-thickening behaviour described by Eq. (4), the nominal stress versus strain relationships at various shear strain-rates could be calculated according to Eqs. (8) - (24). However, for the non-Newtonian fluids whose deformation covers both shear thinning and shear thickening regimes, e.g. the presently studied STF as shown in Fig. 1, it is difficult to integrate Eq. (17) with the complex boundary between shear thinning and shear thickening, and the dynamic compression behaviour of the STF cannot be analytically given by this model. Therefore, the coupled EulerianLagrangian FSI simulation method, where the Eulerian domains are overlapping with the Lagrangian domain [42, 44], is employed to analyse the dynamic squeezing behaviour of the STF. The schematic of the simulation model based on the experimental setup is shown in Fig. 3(a). The loading bar with a size of $\Phi 19 \times 1800 \mathrm{~mm}$ is attached by a trumpet-shaped end with a thickness of $30 \mathrm{~mm}$ and a maximum diameter of $75 \mathrm{~mm}$. The cylindrical shape of the STF specimen with a size of $\Phi 52 \times 9.4 \mathrm{~mm}$ surrounded by the air is placed between the trumpet-shaped end and a rigid body. The air domain is also modelled to ensure the STF could be squeezed into the surrounding air during the compression. The arbitrary Lagrangian-Eulerian (ALE) algorithm [45, 46] by using the constrained model *CONSTRAINED_LAGRANGE_IN_SOLID, which is implemented by LS-DYNA [42], is employed to

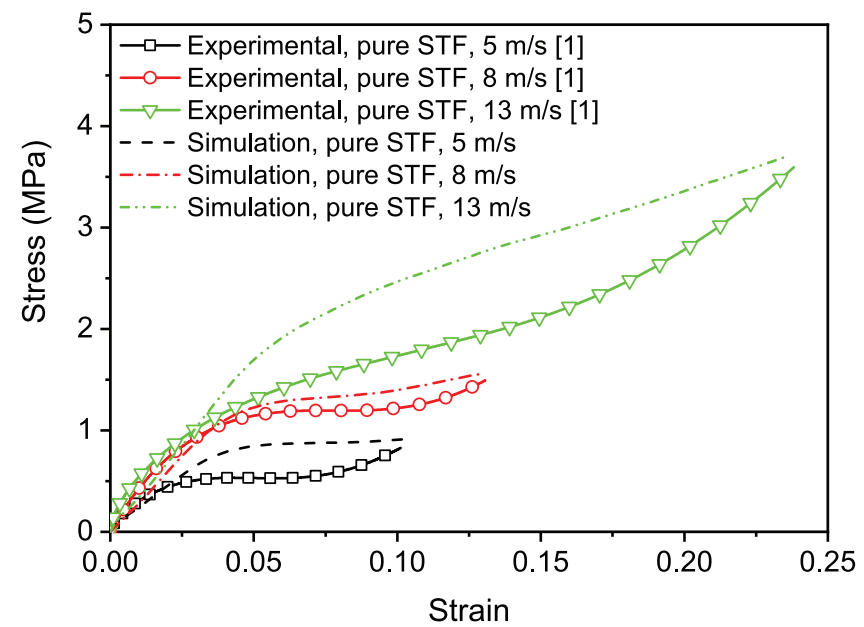

Fig. 4. Analytical stress versus strain relationships of the pure STF at various compressive velocities with the shear-thinning regime parameters $m=16$ and $n=0.47$, and the shear-thickening regime parameters $m=72$ and $n=1.35$. analyse the interaction between the loading end and the STF. The ALE model *ALE_MULTI_MATERIAL_GROUP and the material model *MAT_ALE_VISCOUS are used to simulate the STF behaviour. The ALE algorithm governing equations of mass, momentum and the energy are

$\left(\frac{\partial \rho}{\partial t}\right)_{\kappa}=-\rho \frac{\partial v_{i}}{\partial x_{i}}-c_{i} \frac{\partial \rho}{\partial x_{i}}$

$\rho\left(\frac{\partial v_{i}}{\partial t}\right)_{\kappa}=\frac{\partial \sigma_{i j}}{\partial x_{j}}+\rho f_{i}-\rho c_{i} \frac{\partial v_{i}}{\partial x_{j}}$

$\left(\rho \frac{\partial e}{\partial t}\right)_{\kappa}=\sigma_{i j} \frac{\partial v_{i}}{\partial x_{j}}-\frac{\partial q_{i}}{\partial x_{i}}-\rho c_{i} \frac{\partial e}{\partial x_{i}}$,

where $\rho$ is density of the material, $v_{i}$ is velocity in Lagrange coordinate, $\sigma_{i j}$ is Cauchy stress tensor, $f_{i}$ is body force in unit mass, $c_{i}$ is convection velocity, $q_{i}$ is heat flux, and $e$ is internal energy [44-47].

In order to prevent the leakage in FSI simulation, the mesh sizes of the Eulerian elements are smaller than the Lagrangian elements on the coupling boundaries. The loading bar and the rigid body are fine-meshed with 296,892 hexahedral Lagrangian elements, and the STF and the surrounding air are meshed with 203,776 hexahedral Eulerian elements. In each Lagrangian element, two coupling points are used to increase the simulation accuracy. The domains of the STF and the air are meshed with the share of boundary nodes to ensure the continuity of displacement, velocity and acceleration. The acceleration and velocity at the fluid-structure boundaries are constrained. The compression, tension, and shear directions are coupled to simulate the sticky behaviour of the STF. The Mie-Grüneisen EOS as given in Section 2 is used for the STF. It can be re-written as

$P=\frac{\rho_{0} C_{0}^{2} \mu_{0}\left(1+\left(1-\frac{\gamma_{0}}{2} \mu_{0}\right)-\frac{a}{2} \mu_{0}^{2}\right)}{\left(1-\left(S_{1}-1\right) \mu_{0}-S_{2} \frac{\mu_{0}^{2}}{\mu_{0}+1}-S_{3} \frac{\mu_{0}^{3}}{\left(\mu_{0}+1\right)^{2}}\right)^{2}}+\left(\gamma_{0}+a \mu_{0}\right) E$,

where $\mu_{0}=\frac{\rho}{\rho_{0}}-1, C_{0}$ is intercept of the $U_{S}-U_{P}$ curve, $S_{1}, S_{2}, S_{3}$ are coefficients of the slope of the $U_{S}-U_{P}$ curve, $\gamma_{0}$ is Mie-Grüneisen constant, $a$ is first order volume correction to $\gamma_{0}, \rho$ is current density, and $\rho_{0}$ is original density [48]. The related constitutive parameters of the STF are listed in Table 1. It should be noted that the Mie-Grüneisen EOS does not describe the formation and collapse of cavitation bubbles of fluids. However, for fluids with extremely high viscosity such as the

Table 1

Mie-Grüneisen EOS parameters for STF [22,23].

\begin{tabular}{lllllll}
\hline$\rho_{0}\left(\mathrm{~kg} \cdot \mathrm{m}^{-3}\right)$ & $C_{0}\left(\mathrm{~m} \cdot \mathrm{s}^{-1}\right)$ & $S_{1}$ & $S_{2}$ & $S_{3}$ & $\gamma_{0}$ & $a$ \\
\hline 1600 & 2050 & 5.324 & 0 & 0 & 2 & 0
\end{tabular}


Table 2

EOS parameters for air [49].

\begin{tabular}{lcccccccc}
\hline$\rho_{0}\left(\mathrm{~kg} \cdot \mathrm{m}^{-3}\right)$ & $C_{0}$ & $C_{1}$ & $C_{2}$ & $C_{3}$ & $C_{4}$ & $C_{5}$ & $C_{6}$ & $E\left(\mathrm{~J} \cdot \mathrm{m}^{-3}\right)$ \\
\hline 1.29 & 0 & 0 & 0 & 0 & 0.4 & 0.4 & 0 & $2.5 \times 10^{5}$ \\
\hline
\end{tabular}

STF in the present study, the cavitation is very difficult to occur. In addition, the STF will act as solid after the occurrence of the shear thickening behaviour, making it possible to use Mie-Grüneisen EOS for describing the compressibility behaviour of the STF.

The hydrostatic behaviour of the air is described by the linear polynomial EOS,

$P=C_{0}+C_{1} \mu_{0}+C_{2} \mu_{0}^{2}+C_{3} \mu_{0}^{3}+\left(C_{4}+C_{5} \mu_{0}+C_{6} \mu_{0}^{2}\right) E$,

where $\mu_{0}=\frac{\rho}{\rho_{0}}-1, C_{0} \sim C_{6}$ are parameters, $E$ is internal energy, $\rho$ is current density, and $\rho_{0}$ is original density [49].

The related constitutive parameters of the air are listed in Table 2.

As shown in Fig. 3(b), the loading pressure histories at the impact velocities of 5,8 , and $13 \mathrm{~m} / \mathrm{s}$ measured in previous experiments [1], are applied on the cross-section of the left end of the loading bar. The same data processing method used in previous experiments [1] is employed to obtain the nominal stress versus strain relationship of the STF.

The hydrodynamic constitutive model in Section 2 is employed for the STF. Both shear thinning and shear thickening regimes are taken into account in the numerical simulation. As shown in Fig. 1, the shearthinning behaviour of the STF between the shear strain-rates $5 \times 10^{-2}$ to $25 \mathrm{~s}^{-1}$ in the numerical model is fitted according to the rheology test results,

$\mu_{i j}=16 \cdot \dot{\varepsilon}_{i j}^{0.47-1}$.

It has mentioned that the viscosity of the STF in the shear-thickening regime could not be fully measured at high shear strain-rates due to the limitation of the rotational rheometer. As a result, the shearthickening behaviour of the STF is determined by comparing the numerical simulation results with various thickening parameters and the dynamic compression experimental results. It is found that with shear thickening behaviour of

$\mu_{i j}=72 \cdot \dot{\varepsilon}_{i j}^{1.35-1}$,

the stress versus strain relationships of the STF based on FSI model as given in Fig. 4 are a little higher than their measured stresses [1] when the strains are larger than $2.2 \%, 4.1 \%$ and $3.1 \%$ for impact velocities of 5,8 and $13 \mathrm{~m} / \mathrm{s}$, respectively. We have performed a series of simulations with various shear-thickening parameters and find that $m=72$ and $n=1.35$ in the shear-thickening regime gives the best results. It will be shown later that, with these shear-thickening parameters, the numerical simulated compressive strength of the SPLTC-STF also agrees with the experimental results. Therefore, $m=16$ and $n=0.47$ in the shear-thinning regime and $m=72$ and $n=1.35$ in the shear-thickening regime are used for the non-Newtonian viscosity parameters of the STF. When $\dot{\varepsilon}_{i j}<25 \mathrm{~s}^{-1}$, Eq. (30) is used, and when $\dot{\varepsilon}_{i j}>31 \mathrm{~s}^{-1}$, Eq. (31) is used to describe the viscosity of the STF. When $25<\dot{\varepsilon}_{i j}<31 \mathrm{~s}^{-1}$, the linear relationship between the logarithmic of viscosity and the logarithmic of shear strain-rate is used to ensure the continuity of computation. Fig. 5(a) shows the squeezing stress versus strain relationships of the STF with various shear-thickening constants $m$ and $n$ under the impact velocity of $8 \mathrm{~m} / \mathrm{s}$, and the continuous transition from shearthinning to shear-thickening behaviours is shown in Fig. 5(b). It is clearly shown that the squeezing stress of the STF increases significantly with the increase of viscosity.

\section{Plastic buckling behaviour of SPLTC-STF}

The dynamic compressive response involving elastic buckling and post plastic buckling of core struts of SPLTC-STF is analysed with the same FSI method [42] as the squeezing model of non-Newtonian fluids. The loading methods, as well as the data processing procedures for the SPLTC, the SPLTC filled with water (SPLTC-WT) and the SPLTC-STF, are the same as the squeezing model of non-Newtonian fluids as shown in the Fig. 3(a). The pressure histories at various impact velocities measured in the previous experiments [1] are applied on the crosssection of the left end of the loading bar. The numerical model for the SPLTC with the same dimensions as the previous experiments [1] is built, as shown in Fig. 6(a). The upper surface is loaded; the lower surface of the bottom panel is fully constrained; the rest sides have free boundary conditions. The FSI numerical models for the SPLTC-WT and the SPLTC-STF are developed by adding water or STF inside the inner space of the SPLTC model and air in the surrounded domain with a nonreflecting boundary as shown in Fig. 6(b). The same boundary conditions are applied on the lower surface and the rest sides of the SPLTCWT or the SPLTC-STF as in the model of the SPLTC. The ALE algorithm $[45,46]$ as used in Section 3 is employed to analyse the interaction between the SPLTC and the STF or water filler. The mesh sizes of the Eulerian elements in the model of the SPLTC-WT and SPLTC-STF are smaller than the Lagrangian elements to prevent leakage of fluids. The loading bar and the SPLTC are meshed with 292,560 and 183,600 hexahedral Lagrangian elements, and the surrounded air, the filled water or STF are meshed with 1472,480 and 549,280 hexahedral Eulerian elements, respectively. In each Lagrangian element, two coupling points are used to increase the simulate accuracy. The elements of the air and the filled water or STF share the fluid boundary nodes to ensure continuity of displacement, velocity, and acceleration. The acceleration and velocity at the structure-fluid boundaries are constrained. The compression, tension, and shear directions are coupled to simulate the sticky behaviour of the STF.

The mechanical behaviour of the sandwich panels made of 304 stainless steel is described by the Johnson-Cook constitutive model and the Mie-Grüneisen EOS [16,50]. The effective stress is described as

$\sigma_{y}=\left(A+B \bar{\varepsilon}^{n}\right)\left(1+C \ln \dot{\varepsilon}^{*}\right)$,

where $\overline{\varepsilon^{p}}$ is the equivalent plastic strain; $\dot{\varepsilon}^{*}$ is the normalized equivalent plastic strain-rate; $A, B$, and $C$ are material parameters; $n$ is the work hardening exponent. The hydrostatic behaviour of the STF, the SPLTC, and the water are described by the Mie-Grüneisen EOS [6,22,23,48], and the hydrostatic behaviour of the air is described by the linear polynomial EOS [49]. The related constitutive parameters are given in Tables 1-5.

As shown in Fig. 7(a), the loading pressure histories at the impact velocities of 5, 10 and $16 \mathrm{~m} / \mathrm{s}$ measured in previous experiments [1] are applied on the cross-section of the left end of the loading bar. Firstly, the mesh sensitivity of the numerical model is performed. Fig. 7(b) gives the simulated nominal compressive stress versus strain relationships of the SPLTC model and the SPLTC-STF model meshed with different element numbers at the impact velocity of $10 \mathrm{~m} / \mathrm{s}$. It could be seen that the numerical simulation result of the SPLTC model meshed with 476,120 Lagrangian elements at the impact velocity of $10 \mathrm{~m} / \mathrm{s}$ is almost the same as that meshed with 1761,320 Lagrangian elements. In addition, the numerical simulated result of the SPLTC-STF model meshed with 476,120 Lagrangian elements and 2021,760 Eulerian elements at the impact velocity of $10 \mathrm{~m} / \mathrm{s}$ is almost identical to that meshed with 476,120 Lagrangian elements and 2949,120 Eulerian elements. Therefore, the mesh strategies of the numerical models satisfy the condition of convergence, and the SPLTC model meshed with 476,120 Lagrangian elements and the SPLTC-STF model meshed with 476,120 Lagrangian elements and 2021,760 Eulerian elements are used in the following numerical simulations.

Fig. 8(a) shows the numerically predicted nominal stress versus strain relationships of the SPLTC at the impact velocities of 5 and $10 \mathrm{~m} /$ $\mathrm{s}$ and of the SPLTC-WT at the impact velocity of $16 \mathrm{~m} / \mathrm{s}$. The previous 


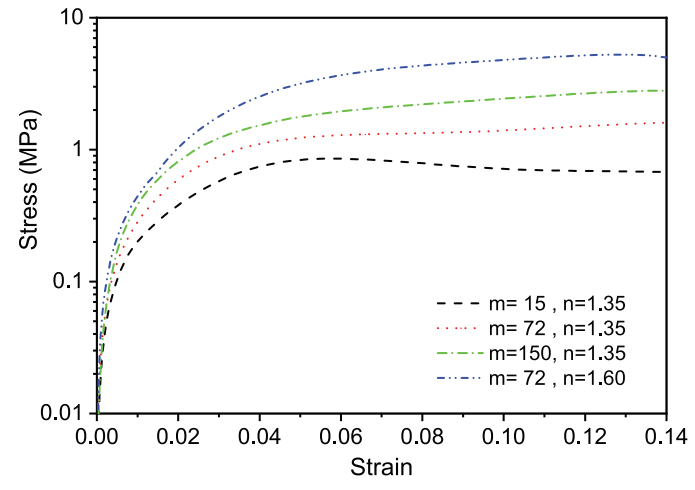

(a)

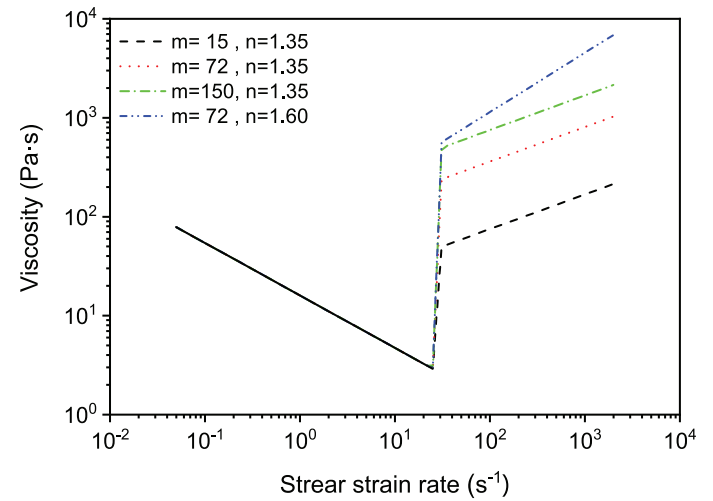

(b)

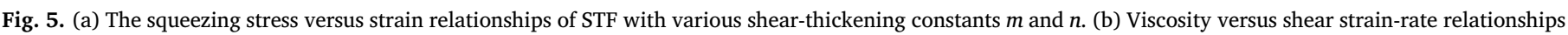
of STF with various shear-thickening regime constants $m$ and $n$.

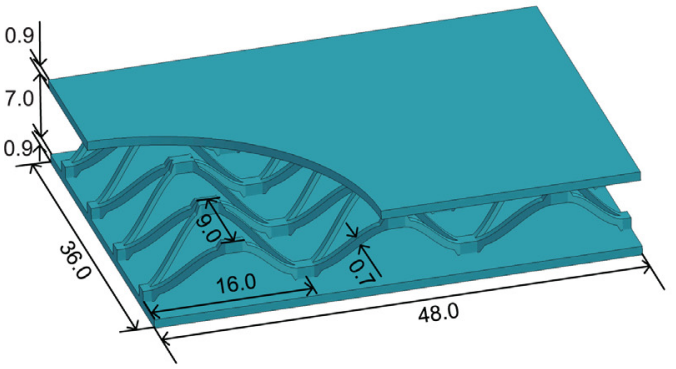

All dimension in $\mathrm{mm}$

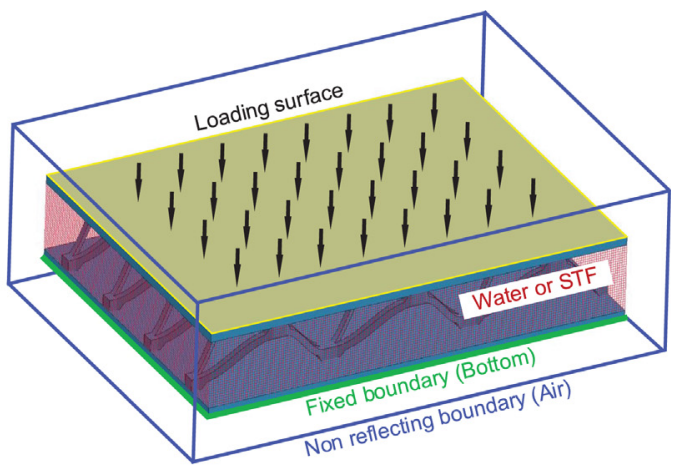

(b)

(a)

Fig. 6. Numerical models of (a) SPLTC, and (b) SPLTC-WT or SPLTC-STF.

Table 3

Material properties for 304 stainless steel [6].

\begin{tabular}{llllll}
\hline$E(\mathrm{GPa})$ & Poisson's ratio & $A(\mathrm{MPa})$ & $B(\mathrm{MPa})$ & $C$ & $n$ \\
\hline 200 & 0.33 & 310 & 1000 & 0.064 & 0.34 \\
\hline
\end{tabular}

Table 4

EOS parameters for 304 stainless steel [6].

\begin{tabular}{lllllll}
\hline$\rho_{0}\left(\mathrm{~kg} \cdot \mathrm{m}^{-3}\right)$ & $C_{0}\left(\mathrm{~m} \cdot \mathrm{s}^{-1}\right)$ & $S_{1}$ & $S_{2}$ & $S_{3}$ & $\gamma_{0}$ & $a$ \\
\hline 7800 & 4570 & 1.49 & 0 & 0 & 0.34 & 0.46 \\
\hline
\end{tabular}

Table 5

EOS parameters for water [48].

\begin{tabular}{lllllll}
\hline$\rho_{0}\left(\mathrm{~kg} \cdot \mathrm{m}^{-3}\right)$ & $C_{0}\left(\mathrm{~m} \cdot \mathrm{s}^{-1}\right)$ & $S_{1}$ & $S_{2}$ & $S_{3}$ & $\gamma_{0}$ & $a$ \\
\hline 1000 & 1647 & 2.56 & 1.986 & 1.2268 & 0.5 & 0 \\
\hline
\end{tabular}

related experimental results of the SPLTC and the SPLTC-WT [1] are also given. It can be seen that the numerically simulated results agree well with the experimental results, validating the numerical models of the SPLTC and the SPLTC-WT. Fig. 8(b) shows the simulated and experimental deformation behaviour of the SPLTC after dynamic compression at the impact velocity of $5 \mathrm{~m} / \mathrm{s}$. The numerical models could also well predict the non-symmetrical deformation behaviour of the SPLTC and the SPLTC-WT, as observed in experiments [1].
The simulated nominal compressive stress versus strain relationships of the SPLTC-STF at impact velocities of 5, 10, and $16 \mathrm{~m} / \mathrm{s}$ are given in Fig. 9(a), and the related experimental results [1] are also given for comparison. Similar to the experimental observation, the simulated nominal compressive stress of the SPLTC-STF initially increases almost linearly with the increase of strain. Then it continues to increase slowly with further compression even after the attainment of the buckling of core struts. The simulated results of the SPLTC-STF agree with the experimental results except for the higher stiffness of the simulated results, which might be attributed to the error while determining the viscosity of the STF in the shear-thickening regime. It is very difficult to directly measure the viscosity of STF at high shear strain-rates, which will be further studied in the near future. Fig. 9(b) shows the stress distributions of the SPLTC and the SPLTC-STF in the loading direction after compression at the impact velocity of $5 \mathrm{~m} / \mathrm{s}$, respectively. After undergoing elastic deformation, the struts on one side of the SPLTC begin to buckle and the struts on the other side begin to rotate, leading to the attainment of the peak stress. During the buckling process of the SPLTC, only one side of struts generates large supporting forces. For the SPLTC-STF, after the elastic deformation, the core struts on both sides begin to buckle, and both sides of the struts generate large supporting forces, as shown in Fig. 9(b).

The effective stress distributions in the middle section of the STF of the SPLTC-STF at the strain of $0.002 \%$ and $13.6 \%$ are given in Figs. 10(a) and (b), respectively. As shown in Fig. 10(a), when the core struts begin to deform under the compression, the lateral velocities of the struts give rise to the increase of the viscosity of the surrounding STF. In response, the STF provides the supporting forces against the 


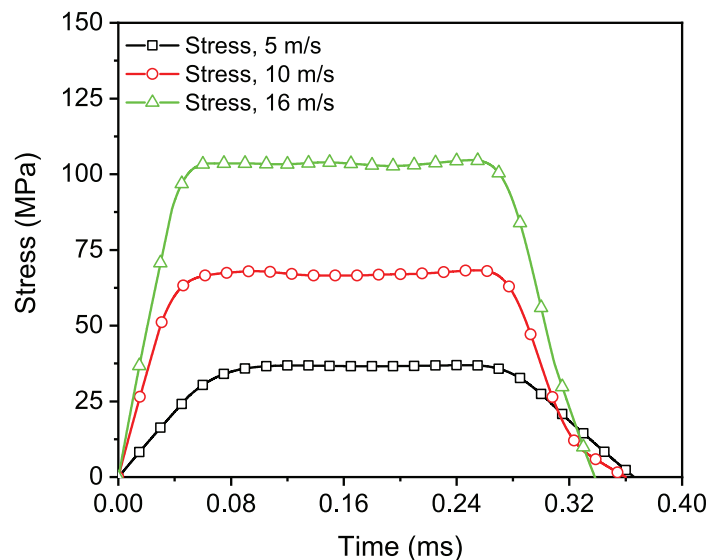

(a)

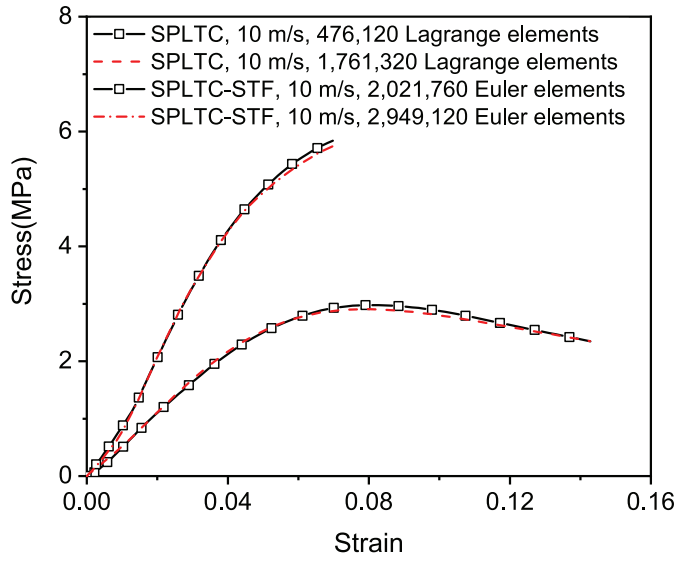

(b)

Fig. 7. (a) Loading pressure histories at the impact velocities of 5, 10, and $16 \mathrm{~m} / \mathrm{s}$. (b) Mesh sensitivity of the numerical models.

lateral movement of the core struts. It should be noted that the ambient atmospheric pressure is not taken into account in the simulation. Even if the atmospheric pressure is taken into account, the counterforces provided by the STF as depicted in Fig. 10(a) is, however, negligible to affect the deformation modes of the core struts, showing that the FSI interaction between the STF filler and the core struts during its buckling is not the reason to transform the deformation modes from asymmetry to symmetry of the SPLTC after being filled with STF. As shown in Fig. 10(b), with further compression, the effective stress of the STF near the periphery of the SPLTC-STF increases significantly. Correspondingly, the large symmetrically distributed outward counterforces are applied on the core struts in the periphery of the STF, which prevented the asymmetrical deformation-induced lateral movement of the upper panel of the SPLTC, as shown in Fig. 8(b). Therefore, the lattice core struts of the SPLTC-STF are forced to deform symmetrically as shown in Fig. 9(b) and consequently significantly improve the dynamic energy absorption capacity of the SPLTC-STF. Actually, the large outward counterforces provided by the STF near the periphery of the SPLTC-STF are generated by the strong interaction between the core struts and the fast flow STF. As shown in Fig. 11, the outwards flow velocities of the STF near the periphery of the SPLTC-STF increase quickly with the increase of strain. When it flows across the core struts with fast velocity, the STF is sheared under high shear strain-rates, leading to the shear thickening of the STF and the high amplitude counterforces on the core struts. The interaction mechanism between the SPLTC and the STF

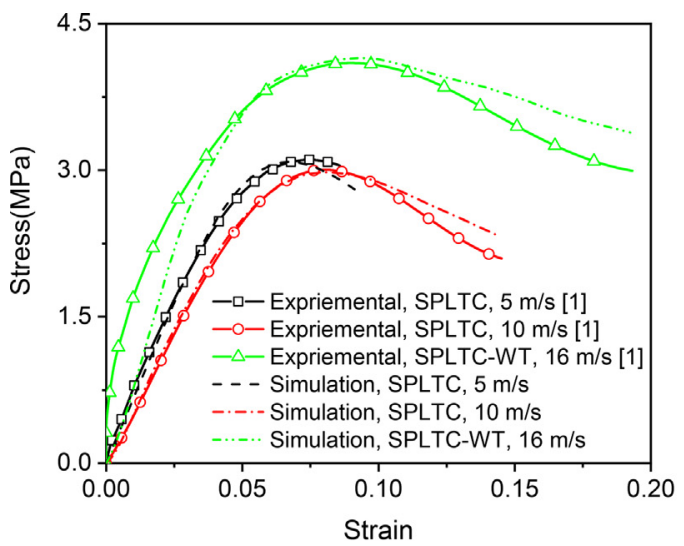

(a) proposed in the previous study [1] needs to be revisited.

The simulated nominal compressive behaviour of the pure STF and the SPLTC at the impact velocity of $5 \mathrm{~m} / \mathrm{s}$, and of the SPLTC-STF and SPLTC-WT at the impact velocity of $16 \mathrm{~m} / \mathrm{s}$ are given in Fig. 12(a). As a comparison, a 'total' stress-strain relationship calculated by directly adding the stress-strain curves of the pure STF and the SPLTC at the impact velocity of $5 \mathrm{~m} / \mathrm{s}$ is also given in Fig. 12(a). The numerical simulated specific energy absorption versus strain relationships the SPLTC at the impact velocity of $10 \mathrm{~m} / \mathrm{s}$, and the SPLTC-STF and the SPLTC-WT at the impact velocity of $16 \mathrm{~m} / \mathrm{s}$ are given in Fig. 12(b). The dynamic stress of the SPLTC-STF at the strain of $4.5 \%$ is about $60 \%$ and $35 \%$ higher than those of the SPLTC at the impact velocity of $5 \mathrm{~m} / \mathrm{s}$ and the SPLTC-WT at the impact velocity of $16 \mathrm{~m} / \mathrm{s}$, respectively. In addition, the numerical simulated peak strength of the SPLTC-STF is much higher than the 'total' strength of the pure STF and the SPLTC, and the numerical simulated specific energy absorption of SPLTC-STF is much higher than the specific energy absorption of SPLTC and SPLTC-WT after the strain of $6.1 \%$, showing the enhanced dynamic energy absorption capacity of the SPLTC-STF as observed in the previous experiments [1].

The nominal compressive responses of the SPLTC filled with nonNewtonian or Newtonian fluids with various viscosities at the impact velocity of $10 \mathrm{~m} / \mathrm{s}$ are shown in Fig. 13. The stress at the same strain of the sandwich panels increases with increasing viscosity of the fluid filler. Unlike the rapid decrease of the compressive stress after

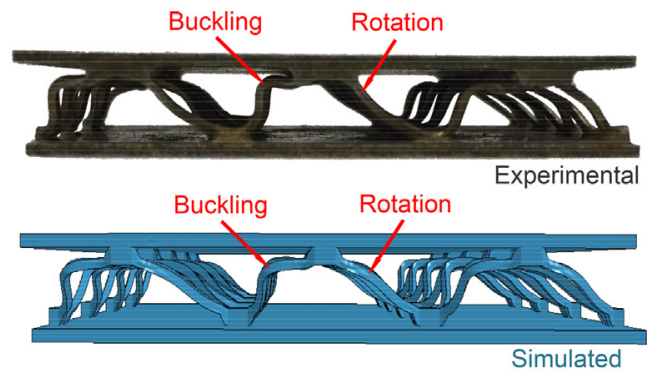

(b)

Fig. 8. (a) Simulated nominal stress versus strain relationships for SPLTC at impact velocities of 5 and $10 \mathrm{~m} / \mathrm{s}$, and for SPLTC-WT at the impact velocity of $16 \mathrm{~m} / \mathrm{s}$. (b) Deformation behaviour of the SPLTC after dynamic compression at the impact velocity of $5 \mathrm{~m} / \mathrm{s}$. 


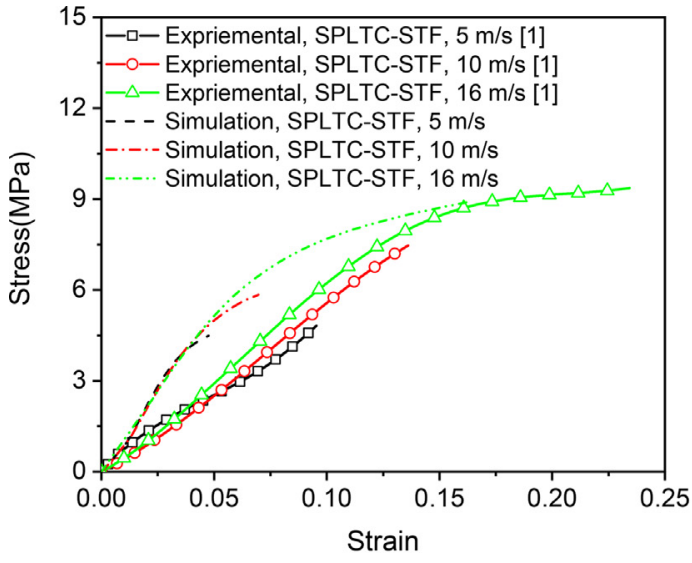

(a)

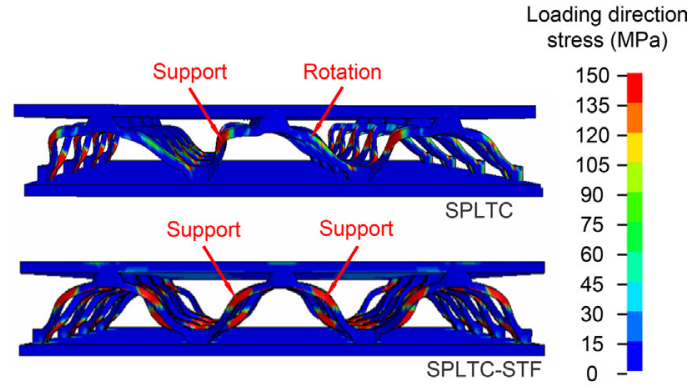

(b)

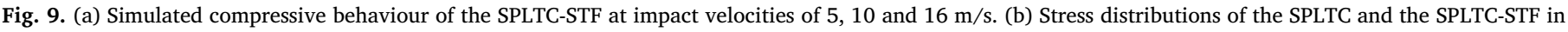
loading direction after compression at the impact velocity of $5 \mathrm{~m} / \mathrm{s}$.

attainment of the buckling of core struts of the SPLTC or the SPLTC-WT [1], the nominal compressive stresses of the SPLTC-STF and the SPLTC filled with fluids with constant high viscosity continue to increase after the viscosities exceed a certain value, by which method the energy absorption capacity of the sandwich panels could be increased significantly. The energy absorption capacity of the sandwich panels could be optimized by filling STF with different shear thickening behaviour. However, it is worth emphasizing that only the shear thinning and shear thickening regimes are taken into account in the material constitutive model of the STF. Actually, the solid-state STF may experience failure behaviours such as crack and yield under relatively high pressure as observed by Waitukaitis and Jaeger [16]. The failure behaviour of the solid-like STF in the shear-thickening regime, which will limit the shear stress of the STF, could be induced during fast compression. In the future, we will try to measure the dynamic behaviour of the solid-like STF in the shear-thickening regime to give a complete description of the constitutive model of the STF in a wide range of loading condition.

\section{Conclusions}

In this paper, the dynamic behaviour of the SPLTC-STF at high strain-rates is studied by modelling and numerical simulation. The main conclusions are as follows.

1 A hydrodynamic constitutive model for STF is employed to describe the complex dynamic behaviour involving shear thinning, shear thickening, and bulk compressibility, and the related constitutive
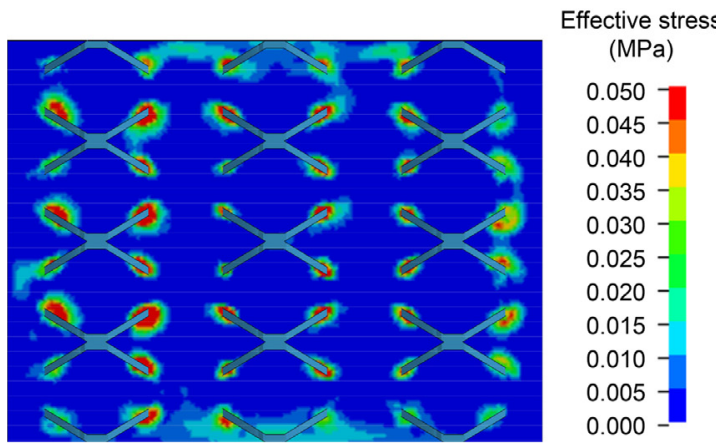

(a) model parameters are obtained by experiments and numerical simulation.

2 An analytical model is developed for describing the dynamic squeezing behaviour of non-Newtonian fluids, which is used to determine the viscosity of STF in shear thickening regime indirectly through the calibration with squeezing experimental results.

3 The strong interaction between the SPLTC and the STF during the fast flow of the STF near the periphery is obtained, which leads to the transformation of deformation mode and therefore contributes to the enhanced energy absorption capacity of the SPLTC-STF.

4 The effects of shear thickening behaviour on the dynamic response of the SPLTC-STF are obtained by the FSI simulation. The results show that the energy absorption capacity of the SPLTC-STF increases with increasing viscosity of the fluid. The hydrodynamic constitutive model for STF and the coupling effects between the STF and lattice structures with various topologies will be investigated in the near future.

\section{CRediT authorship contribution statement}

Z.P. Gu: Methodology, Formal analysis, Writing - original draft. X.Q. Wu: Conceptualization, Writing - review \& editing, Funding acquisition. Q.M. Li: Investigation, Writing - review \& editing. Q.Y. Yin: Resources, Validation. C.G. Huang: Supervision, Project administration.

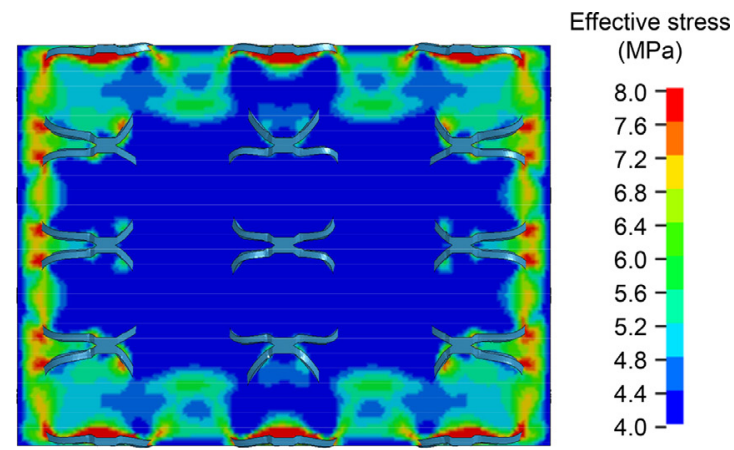

(b)

Fig. 10. Effective stress distributions in the middle section of the STF at the strain of (a) $0.002 \%$ and (b) $13.6 \%$ at the impact velocity of $16 \mathrm{~m} / \mathrm{s}$. 


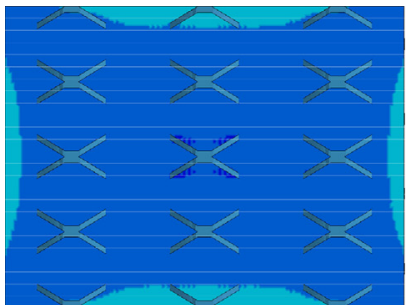

Strain of $0.5 \%$

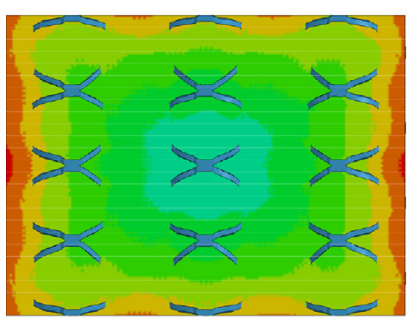

Strain of $9.6 \%$

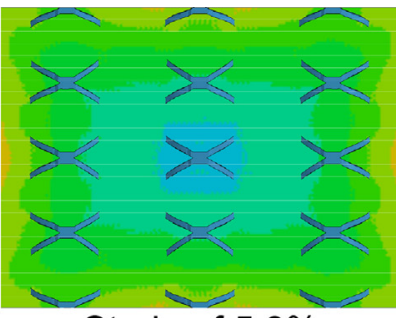

Strain of $5.0 \%$

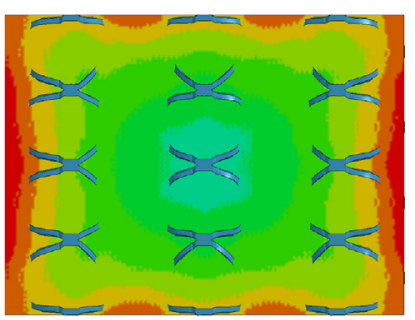

Strain of $13.6 \%$

\section{Resultant velocity}

$(\mathrm{m} / \mathrm{s})$

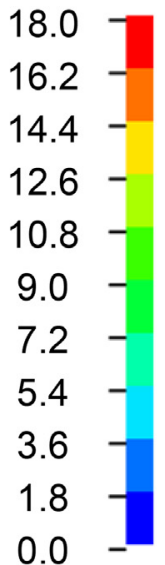

Fig. 11. Flow velocity distributions in the middle section of the STF at the strain of $0.5 \%, 5.0 \%, 9.6 \%$ and $13.6 \%$ at the impact velocity of $16 \mathrm{~m} / \mathrm{s}$.

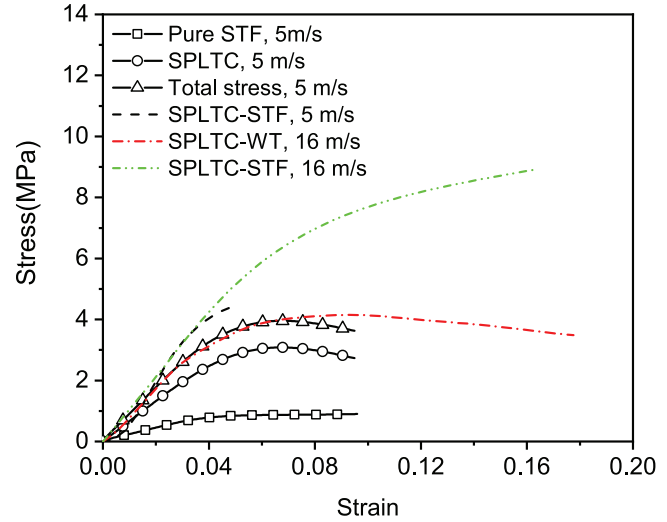

(a)

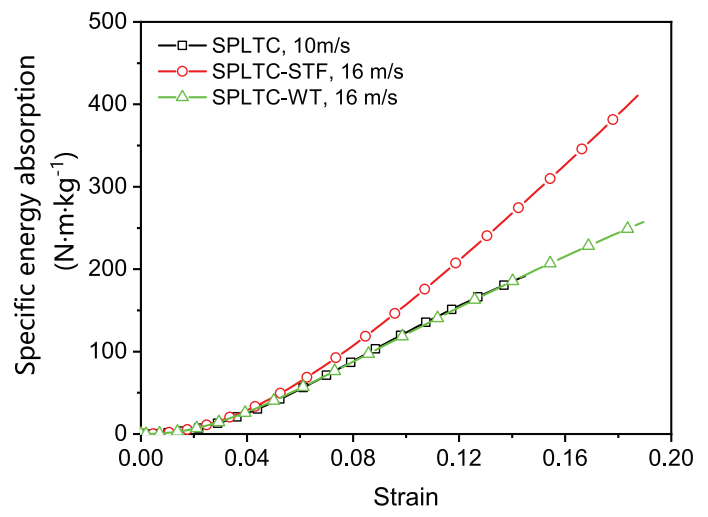

(b)

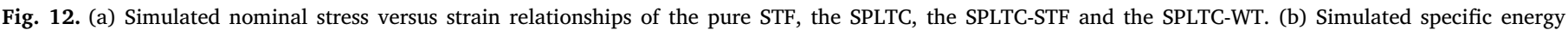
absorption versus strain relationships the SPLTC, the SPLTC-STF and the SPLTC-WT.

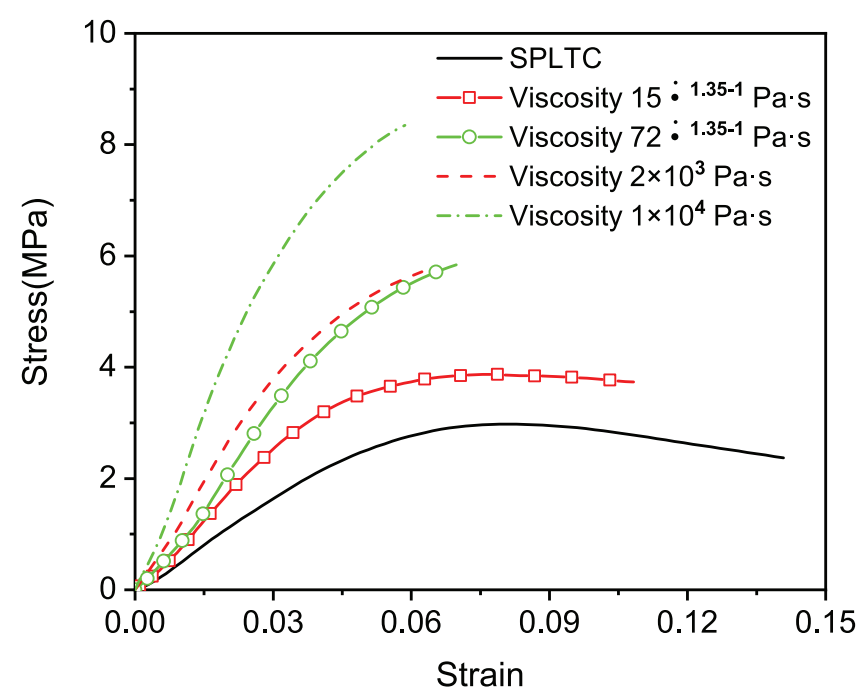

Fig. 13. Nominal compressive stress versus strain relationships of the SPLTCSTF and the SPLTC filled with Newtonian fluids with constant viscosities at the impact velocity of $10 \mathrm{~m} / \mathrm{s}$.

\section{Declaration of Competing interests}

The authors declare that they have no known competing financial interests or personal relationships that could have appeared to influence the work reported in this paper.

\section{Acknowledgements}

This research was supported by the National Natural Science Foundation of China (Grant Nos. 11672315, 11772347 and 11802349), the Science Challenge Project (Grant No. TZ2018001), and the Strategic Priority Research Program of the Chinese Academy of Sciences (Grant Nos. XDB22040302 and XDB22040303).

\section{Supplementary materials}

Supplementary material associated with this article can be found, in the online version, at doi:10.1016/j.ijimpeng.2020.103616.

\section{References}

[1] Wu X, Xiao K, Yin Q, et al. Experimental study on dynamic compressive behaviour of sandwich panel with shear thickening fluid filled pyramidal lattice truss core. Int J Mech Sci 2018;138. 467-75. 
[2] Evans AG, Hutchinson JW, Fleck NA, et al. The topological design of multifunctional cellular metals. Prog Mater Sci 2001;46(3-4). 309-27.

[3] Hutchinson JW, Xue ZY. Metal sandwich plates optimized for pressure impulses. Int J Mech Sci 2005;47(4-5). 545-69.

[4] Wadley HNG. Multifunctional periodic cellular metals. Philos T Roy Soc A 2006;364(1838):31-68.

[5] Deshpande VS, Fleck NA. One-dimensional response of sandwich plates to underwater shock loading. J Mech Phys Solids 2005;53(11). 2347-83.

[6] Lee S, Barthelat F, Hutchinson JW, et al. Dynamic failure of metallic pyramidal truss core materials - Experiments and modeling. Int J Plasticity 2006;22(11). 2118-45.

[7] McShane GJ, Deshpande VS, Fleck NA. Underwater blast response of free-standing sandwich plates with metallic lattice cores. Int J Impact Eng 2010;37(11). 1138-49.

[8] Mohr D. Mechanism-based multi-surface plasticity model for ideal truss lattice materials. Int J Solids Struct 2005;42(11-12). 3235-60.

[9] Wadley HNG, Borvik T, Olovsson L, et al. Deformation and fracture of impulsively loaded sandwich panels. J Mech Phys Solids 2013;61(2). 674-99.

[10] Xue ZY, Hutchinson JW. Constitutive model for quasi-static deformation of metallic sandwich cores. Int J Numer Meth Eng 2004;61(13). 2205-38.

[11] Zhu F, Zhao L, Lu G, et al. A numerical simulation of the blast impact of square metallic sandwich panels. Int J Impact Eng 2009;36(5). 687-99.

[12] Vaziri A, Xue Z, Hutchinson JW. Metal sandwich plates with polymer foam-filled cores. J Mech Mater Struct 2006;1(1):97-127.

[13] Yungwirth CJ, Radford DD, Aronson M, et al. Experiment assessment of the ballistic response of composite pyramidal lattice truss structures. Compos Part B-Ene 2008;39(3). 556-69.

[14] Wadley HNG, Dharmasena KP, O'Masta MR, et al. Impact response of aluminum corrugated core sandwich panels. Int J Impact Eng 2013;62. 114-28.

[15] Jiang W, Gong X, Xuan S, et al. Stress pulse attenuation in shear thickening fluid. Appl phys lett 2013;102(10):101901.

[16] Waitukaitis SR, Jaeger HM. Impact-activated solidification of dense suspensions via dynamic jamming fronts. Nature 2012;487(7406). 205-9.

[17] Bender J, Wagner NJ. Reversible shear thickening in monodisperse and bidisperse colloidal dispersions. J Rheol 1996;40(5):899-916.

[18] Petel OE, Hogan JD. An investigation of shear thickening fluids using ejecta analysis techniques. Int J Impact Eng 2016;93:39-48.

[19] Newstein MC, Wang H, Balsara NP, et al. Microstructural changes in a colloidal liquid in the shear thinning and shear thickening regimes. J Chem Phys 1999;111(10). 4827-38.

[20] Barnes HA. Shear-thickening (dilatancy) in suspensions of nonaggregating solid particles dispersed in newtonian liquids. J Rheol 1989;33(2). 329-66.

[21] Laun HM, Bung R, Hess S, et al. Rheological and small-angle neutron-scattering investigation of shear-induced particle structures of concentrated polymer dispersions submitted to plane poiseuille and couette-flow. J Rheol 1992;36(4). 703-41.

[22] Wu X, Zhong F, Yin Q, et al. Dynamic response of shear thickening fluid under laser induced shock. Appl phys lett 2015;106(7):071903.

[23] Wu X, Yin Q, Huang C. Experimental study on pressure, stress state, and temperature-dependent dynamic behaviour of shear thickening fluid subjected to laser induced shock. J Appl Phys 2015;118(17):173102.

[24] Gurgen S, Sofuoglu MA. Vibration attenuation of sandwich structures filled with shear thickening fluids. Compos Part B-Eng 2020;186:107831.

[25] Fischer C, Braun SA, Bourban PE, et al. Dynamic properties of sandwich structures with integrated shear-thickening fluids. Smart Mater Struct 2006;15(5):1467-75.

[26] Guo Y, Wei Y, Zou J, et al. Impact and usage of the shear thickening fluid (STF) material in damping vibration of bolted flange joints. Smart Mater Struct 2019;28(9):095005

[27] Gurgen S. An investigation on composite laminates including shear thickening fluid under stab condition. J Compos Mater 2019;53(8):1111-22.

[28] Tan ZH, Zuo L, Li WH, et al. Dynamic response of symmetrical and asymmetrical sandwich plates with shear thickening fluid core subjected to penetration loading. Mater Design 2016;94. 105-10.

[29] Xue ZY, Hutchinson JW. A comparative study of impulse-resistant metal sandwich plates. Int J Impact Eng 2004;30(10). 1283-305.

[30] Zhang P, Cheng Y, Liu J, et al. Experimental and numerical investigations on laserwelded corrugated-core sandwich panels subjected to air blast loading. Mar Struct 2015;40. 225-46.

[31] Xue ZY, Hutchinson JW. Crush dynamics of square honeycomb sandwich cores. Int J Numer Meth Eng 2006;65(13). 2221-45.

[32] Wei X, Phuong T, de Vaucorbeil A, et al. Three-dimensional numerical modeling of composite panels subjected to underwater blast. J Mech Phys Solids 2013;61(6). 1319-36.

[33] Tilbrook MT, Deshpande VS, Fleck NA. Underwater blast loading of sandwich beams: regimes of behaviour. Int J Solids Struct 2009;46(17). 3209-21.

[34] Dharmasena KP, Wadley HNG, Williams K, et al. Response of metallic pyramidal lattice core sandwich panels to high intensity impulsive loading in air. Int J Impact Eng 2011;38(5). 275-89.

[35] Schiffer A, Tagarielli VL. One-dimensional response of sandwich plates to underwater blast: fluid-structure interaction experiments and simulations. Int J Impact Eng 2014;71:34-49.

[36] Kumar S, Tripathi PK, Upadhyay SN. On the mass-transfer in Non-Newtonian fluids 1. Transfer from spheres to power law fluids. Lett Heat Mass Trans 1980;7(1):43-53.

[37] Petel OE, Frost DL, Higgins AJ, et al. Formation of a disordered solid via a shockinduced transition in a dense particle suspension. Phys Rev E 2012;85(2):021401.

[38] Petel OE, Higgins AJ. Shock wave propagation in dense particle suspensions. J Appl Phys 2010;108(11):114918.

[39] Meyers MA. Dynamic behavior of materials. Wiley-Interscience; 1994.

[40] Herrmann W. Constitutive equation for dynamic compaction of ductile porous materials. J Appl Phys 1969;40(6). 2490-9.

[41] McQueen RG, Marsh SP. Shock-wave compression of iron-nickel alloys and earths core. J Geophys Res 1966;71(6). 1751-9.

[42] Hallquist JO. LS-DYNA3D theoretical manual. Livermore software technology corporation; 1993.

[43] Kuzma DC. Fluid inertia effects in squeeze films. Appl Sci Res 1968:18(1):15-20.

[44] Pi SJ, Cheng DS, Cheng HL, et al. Fluid-structure-interaction for a steel plate subjected to non-contact explosion. Theor Appl Fract Mec 2012;59(1):1-7.

[45] Barlow AJ, Maire P-H, Rider WJ, et al. Arbitrary Lagrangian-Eulerian methods for modeling high-speed compressible multimaterial flows. J Comput Phys 2016;322. 603-65.

[46] Souli M, Ouahsine A, Lewin L. ALE formulation for fluid-structure interaction problems. Comput Methods Appl Mech Eng 2000;190(5-7). 659-75.

[47] Hu HH, Patankar NA, Zhu MY. Direct numerical simulations of fluid-solid systems using the arbitrary Lagrangian-Eulerian technique. J Comput Phys 2001;169(2). 427-62.

[48] Panciroli R, Abrate S, Minak G, et al. Hydroelasticity in water-entry problems: comparison between experimental and SPH results. Compos Struct 2012;94(2). $532-9$.

[49] Wang Z-L, Li Y-C, Shen RF. Numerical simulation of tensile damage and blast crater in brittle rock due to underground explosion. Int J Rock Mech Min 2007;44(5). 730-8.

[50] Johnson G, Cook W. A constitutive model and data for metals subjected to large strains, high strain rates and high temperatures. Proceedings of the 7th International Symposium on Ballistics, the hague, Netherlands. 1983. 541-7. 